\title{
Emploi des « seniors » et conditions de travail : une étude statistique comparative entre pays d'Europe
}

\author{
Employment of « senior workers » and working conditions : a statistical \\ comparison between European countries \\ Empleo de « seniors " y condiciones de trabajo : Un estudio estadístico \\ comparativo entre países de Europa
}

\section{Céline Mardon et Serge Volkoff}

\section{(2) OpenEdition}

\section{Journals}

\section{Édition électronique}

URL : http://journals.openedition.org/pistes/1740

DOI : $10.4000 /$ pistes. 1740

ISSN : 1481-9384

Éditeur

Les Amis de PISTES

Édition imprimée

Date de publication : 1 mai 2011

Référence électronique

Céline Mardon et Serge Volkoff, «Emploi des « seniors » et conditions de travail : une étude statistique comparative entre pays d'Europe », Perspectives interdisciplinaires sur le travail et la santé [En ligne], 13-1 | 2011, mis en ligne le 01 mai 2011, consulté le 19 avril 2019. URL : http:// journals.openedition.org/pistes/1740 ; DOI : 10.4000/pistes.1740

Ce document a été généré automatiquement le 19 avril 2019.

\section{(c) (i) (9)}

Pistes est mis à disposition selon les termes de la licence Creative Commons Attribution - Pas d'Utilisation Commerciale - Pas de Modification 4.0 International. 


\title{
Emploi des « seniors » et conditions de travail : une étude statistique comparative entre pays d'Europe
}

\author{
Employment of « senior workers » and working conditions : a statistical \\ comparison between European countries \\ Empleo de « seniors " y condiciones de trabajo : Un estudio estadístico \\ comparativo entre países de Europa
}

Céline Mardon et Serge Volkoff

Nous remercions Gérard Cornilleau et Annie Jolivet pour leurs commentaires sur une première version de cet article. Ils ne sont bien sûr pas responsables de défauts actuels dans ce texte.

1 L'emploi des âgés constitue une préoccupation croissante, notamment pour les institutions internationales, pour deux raisons essentielles: d'une part, la structure démographique de la main-d'œuvre se déplace vers les âges élevés, parce que la natalité dans les cohortes correspondantes - les natifs des années 50 ou 60 - a été plus élevée que dans les décennies suivantes, avec des écarts divers selon les pays industrialisés; d'autre part, l'allongement de la vie professionnelle est souvent préconisé pour faire face aux préoccupations concernant le financement des pensions de retraite.

2 En Europe, avec la définition d'une stratégie commune pour l'emploi, l'objectif d'un maintien des «seniors» au travail est devenu explicite (Jolivet, 2002). L'Union européenne s'est dotée de deux objectifs : un taux d'emploi moyen de $50 \%$ pour les personnes de 55 à 64 ans a été affiché lors du Conseil européen de Stockholm en 2001 ; et un relèvement progressif d'environ 5 ans de l'âge moyen de sortie de l'activité lors du Conseil de Barcelone en 2002. Dans les deux cas l'horizon fixé était 2010. À peu près à miparcours, en 2005, date sur laquelle portera l'étude présentée ici, l'Europe était loin d'atteindre cette cible : le taux d'emploi des 55-64 ans était de 42,5\%.

Dans ces objectifs et indicateurs, il est question d'emploi, non de travail, ni de santé, ni de formation. Une vision plus intégrée des facettes du parcours professionnel est pourtant 
nécessaire. Cette approche élargie est d'ailleurs de règle dans les politiques publiques de pays - nordiques notamment - où l'emploi des seniors est élevé (Cornilleau et coll., 2007) ; ces politiques ont entre autres pour buts l'amélioration des conditions de travail pour tous les travailleurs ou spécialement les seniors, la refonte des carrières, la lutte contre les discriminations liées à l'âge dans tous les aspects de la vie professionnelle, la sensibilisation des entreprises et des travailleurs sur ces thèmes, etc. Bien entendu les auteurs observent par ailleurs des liens entre les taux d'emploi et les caractéristiques socio-économiques ou les dispositifs institutionnels des différents pays d'Europe: le recours important au temps partiel en Finlande, au Royaume-Uni et aux Pays-Bas, l'importance des pensions d'invalidité dans les pays scandinaves, une faible progression des salaires à l'ancienneté dans ces mêmes pays, et plus généralement la situation économique du pays, et les politiques budgétaires et monétaires en vigueur.

4 Nous souhaitons ici approfondir la prise en compte des caractéristiques du travail, en faisant appel à nos propres démarches de recherche. Celles-ci, de façon générale, visent à élucider les liens entre âge et conditions de travail, avec les enjeux que cette liaison recouvre en matière de santé au travail et d'élaboration de l'expérience (David et coll., 2001; Gaudart et coll., 2006). Cette démarche incite à rapprocher trois registres de connaissance : les recherches en ergonomie sur le vieillissement au travail (Delgoulet et coll., 2005), qu'il s'agisse d'observations d'activité sur le terrain, ou de connaissances plus fondamentales issues de la physiologie et de la psychologie du travail; les études microdémographiques (Volkoff, 2001), visant à interpréter les processus de sélection de la main-d'œuvre selon l'âge, dans une entreprise, un atelier, un service, en lien avec les caractéristiques du travail ; et les analyses démographiques plus globales (Molinié, 1999, 2001a et b; 2003; Pailhé, 2002), qui permettent d'évaluer l'ampleur de ces mêmes mécanismes à l'échelle d'un secteur, d'une profession, d'un pays, ou encore d'une génération.

$5 \quad$ L'hypothèse simple qui relie ces niveaux entre eux est la suivante : les affectations de la main-d'œuvre à tel ou tel âge sont liées aux exigences du travail, qui déterminent en partie la possibilité et le souhait d'accéder à une situation de travail donnée, d'y demeurer, ou de s'en éloigner. Or si, chez les plus âgés, l'alternative entre "y demeurer » et « s'en éloigner » recouvre, comme c'est plausible, l'arbitrage entre maintien en emploi et cessation définitive d'activité, on est en droit de chercher des liens entre ces exigences et l'emploi (ou le non-emploi) des seniors, y compris d'un pays à l'autre. C'est l'objet de la présente étude.

\section{Quelques questions prioritaires}

6 L'observation et la compréhension des processus de sélection impliquant l'âge et les contraintes de travail constituent un thème de recherche ancien, dont le développement se poursuit.

7 Pour en retenir quelques exemples, citons d'abord les études rassemblées par Pacaud (1975) : l'auteure montrait le déplacement d'ouvriers qualifiés vieillissants (charpentiers, maçons, plâtriers, plombiers) vers des travaux d'entretien; ou encore, chez des travailleurs âgés qui se maintiennent sur des travaux lourds, la recherche de tâches où la cadence est plus lente. En analysant les postes occupés par des ouvriers de la construction mécanique pendant cinq années, Desriaux et coll. (1987) ont montré comment se reconstruit sans cesse une surreprésentation des jeunes dans les tâches exigeantes quant 
au rythme et à la précision. Gaillard (1995) a attiré l'attention sur les postes les plus sélectifs dans une petite usine de confection : parmi les "piqueuses de housses ", obligées de respecter des contraintes de temps serrées dans des positions très statiques avec de nombreux incidents à gérer, aucune ne se maintenait sur ce poste après 42 ans. L'analyse la plus complète a été présentée par Gonon (2003) à propos des changements d'unité dans un grand hôpital: le travail posté, certaines contraintes physiques, des difficultés psychiques liées à l'état des patients, l'imprévisibilité dans le travail, mais aussi l'utilisation de technologies nouvelles et la demande de polyvalence attirent et/ou maintiennent des infirmières jeunes (entre autres parce que certaines d'entre elles n'ont guère le choix), alors que ces mêmes caractéristiques apparaissent dissuasives pour beaucoup de leurs aînées qui cherchent et parviennent à s'en écarter.

Ces résultats appellent plusieurs interprétations, articulées entre elles. On peut y voir l'effet de l'évolution de la santé avec l'âge qui ne permettrait plus de tolérer certaines contraintes. Ce peut être aussi une évolution de la performance, plus ou moins liée à la santé, avec des difficultés qui apparaissent pour atteindre l'efficacité requise. Il peut s'agir d'une distribution différenciée des connaissances acquises par des personnels de diverses générations. Ou encore de régulations collectives, où les capacités physiques et les compétences présentes dans une équipe sont mises en commun et « distribuées » dans un souci d'efficience. En outre, on peut évoquer le champ d'action que les travailleurs arrivent à se constituer au fil de leur parcours professionnel, ce qui leur confère davantage de liberté pour négocier ou imposer la prise en compte de leurs préférences, et éviter les situations qu'ils ont repérées comme pénalisantes... pour autant que la structure démographique et le niveau des contraintes de travail dans l'entreprise laissent des marges de manœuvre.

Ce que nous retiendrons ici en tout cas, c'est que ces études locales, et les approches statistiques plus globales déjà citées, attirent l'attention sur des difficultés, particulièrement sensibles chez les âgés, liées à quatre grandes catégories d'exigences (Molinié, 2003) : les postures pénibles, les horaires décalés, une pression temporelle élevée ou des changements rapides de technique et d'organisation.

Notre idée ici sera de reprendre chacune de ces quatre caractéristiques, d'expliquer de façon synthétique, à partir des connaissances disponibles, en quoi elles peuvent être problématiques pour les âgés puis d'interroger leur lien avec l'emploi des seniors - dans différents pays d'Europe, pour cette étude. Pour cela, nous examinerons les réponses à l'enquête européenne sur les conditions de travail (EWCS, voir encadré 1). Ces réponses reflètent la perception des individus sur les conditions dans lesquelles ils travaillent, mais l'intérêt de les examiner n'en est en rien diminué, sachant l'impossibilité d'objectiver entièrement les conditions de travail subies par un individu indépendamment de ses caractéristiques physiques et mentales (Gollac, 1998 ; Volkoff et coll., 2005a). On sait par ailleurs qu'une contrainte de travail peut être plus ou moins bien tolérée selon l'âge (David et coll., 2001 ; Molinié, 2003). Des écarts entre pays existent sans aucun doute aussi $\mathrm{du}$ fait des nécessaires imperfections de traduction du questionnaire d'enquête et des différents modes de collecte des données dans les pays participants, mais aussi des différences des systèmes de santé et des institutions de ces pays, et des différences culturelles pouvant influencer les réponses des travailleurs (Merllié et Paoli, 2001). Sur la base de ces données, nous chercherons à répondre à deux questions principales :

1. Vérifie-t-on, dans l'ensemble de l'Europe, une affectation différentielle selon l'âge, au regard de cette caractéristique? 
13 Nous observerons d'abord la fréquence, dans chaque tranche d'âge, de cette caractéristique ou d'une variable approchante (le choix de ladite " variable approchante » n'est pas indifférent, et il faut que cette variable soit présente dans l'enquête ; on verra que, pour la quatrième "exigence » surtout, la question utilisable amène à déplacer quelque peu l'objet d'étude). Puis nous rééditerons cet examen sur la base d'un modèle de régression multivarié afin de repérer l'existence d'un "véritable » effet d'âge, une fois contrôlées quelques autres variables: sexe, pays, grand secteur, taille d'entreprise, grande catégorie socioprofessionnelle.

14 Sur la foi des résultats déjà évoqués, nous nous attendons à une baisse de la fréquence avec l'âge.

15 2. Y a-t-il un lien statistique entre le taux d'emploi des seniors dans un pays et la fréquence globale de cette caractéristique du travail, dans sa main-d'œuvre?

16 Pour préciser l'analyse nous dissocierons les niveaux atteints dans deux catégories d'âge : les 45 ans et plus, et les moins de 45 ans. Le choix d'un seuil à 45 ans reflète un souci de puissance statistique, qui implique d'éviter un grand déséquilibre entre les effectifs des populations que l'on va comparer. Ce choix est par ailleurs justifié du fait que c'est vers cet âge, même s'il existe bien sûr une grande variabilité interindividuelle, que bien des effets du vieillissement deviennent manifestes, avec l'apparition de difficultés qui y sont liées, notamment au travail (Millanvoye, 1995). Les caractéristiques du travail fragilisantes ou excluantes pour un salarié vieillissant peuvent de plus prendre quelques années avant de produire des effets ; on peut donc considérer qu'une politique dédiée aux seniors sera plus efficace si elle est mise en place en amont des 55 ans, âge à partir duquel sont calculés les taux d'emploi des seniors que l'on prendra en compte dans cet article.

Dans un pays les salariés âgés peuvent être "protégés » d'une contrainte de deux façons : parce qu'elle est d'un niveau globalement modéré dans le pays, ce qui évite l'usure en "protégeant» aussi les jeunes; ou parce qu'elle est concentrée sur les jeunes, ce qui laisse alors pressentir des difficultés pour ces salariés eux-mêmes (surexposés), ou pour les entreprises qui dans certains pays ne pourront maintenir ce mode de gestion de la main-d'œuvre, eu égard aux évolutions démographiques.

Nous classerons donc les pays par fréquence décroissante de la contrainte étudiée, dans l'une et l'autre catégorie d'âge, et nous allons confronter chacun de ces deux classements à une gradation des pays par taux d'emploi des seniors décroissant (encadré 2). Nous présenterons ces résultats sous forme graphique, pour une description générale, puis nous indiquerons la valeur et le seuil de signification d'un coefficient de corrélation des rangs (dit "de Spearman»: retenons que ce coefficient vaut 1 quand les classements comparés sont identiques, -1 quand ils sont inverses, et 0 quand ces classements sont indépendants) entre les deux hiérarchies: celle des taux d'emploi, et celle de la contrainte étudiée. Au moment d'indiquer les résultats des tests statistiques nous ne nous limiterons pas aux seuils usuels de significativité (1\%,5\%) - mais nous indiquerons bien sûr les seuils atteints. Cette option, souvent retenue dans nos travaux, ne vise évidemment pas à avancer des affirmations reposant sur des faits fortuits. Nous souhaitons seulement ne pas risquer d'écarter des résultats qui peuvent donner lieu à une interprétation intéressante eu égard aux connaissances scientifiques disponibles (Chia, 1997 ; Volkoff et coll., 2005a). 
19 Nous nous attendons cette fois à trouver, dans les pays à fort taux d'emploi des seniors, des fréquences plutôt basses pour les contraintes étudiées - en tout cas celles constatées chez les 45 ans et plus -, donc des coefficients de corrélation clairement négatifs.

\section{L'obstacle des postures corporelles difficiles}

De façon générale, les performances physiques maximales diminuent avec l'âge à partir de 30-35 ans, car les systèmes articulaire, musculaire et cardio-respiratoire se modifient peu à peu (Millanvoye, 1993). Cependant, aux âges de la vie professionnelle, la force musculaire se maintient à peu près, alors que la souplesse, en particulier celle du dos ou des genoux, se réduit - en tendance moyenne, avec bien sûr de fortes disparités entre individus. Cela entraîne une gêne pour adopter des postures penchées, accroupies, tordues ou déséquilibrées. Le maintien d'une posture difficile devient alors aussi " coûteux », pour le système cardiovasculaire et musculaire, qu'un effort violent. En ce domaine comme dans tous ceux qui concernent l'évolution de l'organisme avec l'âge, les effets du vieillissement «naturel» (si tant est qu'on puisse le définir) se combinent aux traces de la vie, notamment de la vie professionnelle : une exposition prolongée au port de charges lourdes, par exemple, accroît à terme la prévalence des douleurs lombaires, qui à leur tour rendent les postures penchées plus difficiles.

21 Ces difficultés expliquent en partie les mécanismes de sélection dont on a donné plus haut des exemples. Reste que des salariés âgés occupent effectivement des postes de travail exigeants sur le plan postural sans que leur performance soit nécessairement moindre que celle des jeunes. La diversité interindividuelle, sur laquelle on vient d'insister, joue bien sûr un rôle. Surtout, c'est un des domaines où la connaissance du travail, et la connaissance de son propre corps, peuvent permettre d'adopter des modes opératoires moins coûteux physiquement (Cloutier et coll., 1999; Gaudart, 2000; Chassaing, 2004) ; encore faut-il que la conception du poste de travail, son organisation, et les modalités d'apprentissage le permettent.

Dans l'enquête européenne, la question posée à ce sujet est formulée ainsi : «Voulez-vous me dire dans quelle mesure votre travail principal implique des positions douloureuses ou fatigantes?» Les réponses s'échelonnent en fonction d'une fréquence d'exposition, évaluée par l'enquêté, de "jamais » à « tout le temps ». On retiendra ici les modalités égales ou supérieures à «la moitié du temps ».

L'examen des liens entre postures douloureuses et âge, pour l'ensemble des 25 pays (figure ci-dessous), fait apparaître une relation négative, quoique peu marquée. En fréquence brute, le pourcentage de salariés concernés décroît de $32 \%$ pour les plus jeunes à $25 \%$ pour les plus âgés, mais si l'on retire ces tranches extrêmes - et moins peuplées la tendance n'est pas nette. En cotes relatives après la prise en compte d'autres variables, on constate une légère hausse entre 45 et 54 ans, puis une baisse ensuite. 
Figure 1. Postures difficiles au moins la moitié du temps, selon l'âge (salariés de l'UE des 25, 2005)

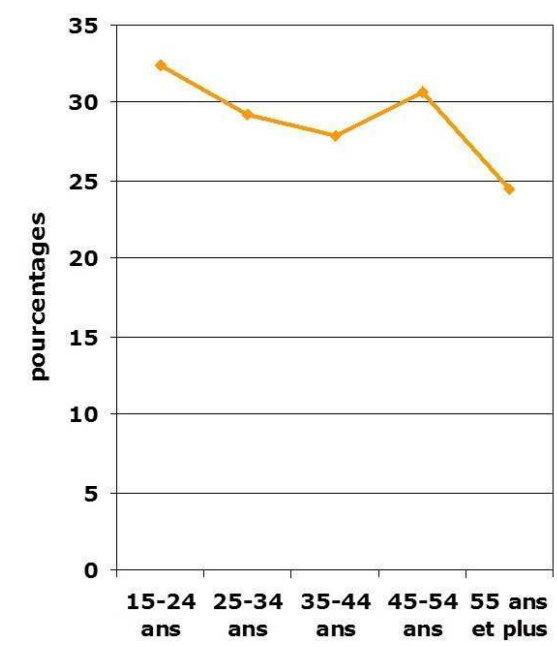

Odds ratios sur l'âge

(autres variables "explicatives»: sexe, pays, grands secteurs, taille entreprise, grandes catégories socioprofessionnelles)

L'analyse par pays (figure suivante - voir en fin d'encadré 2 la signification des abréviations pour chaque nom de pays) confirme d'abord le résultat précédent, en ce sens que les pays qui « protègent » les travailleurs âgés des postures pénibles (par rapport aux jeunes du même pays) ne sont pas plus nombreux que ceux qui au contraire les " surexposent "; on le vérifie en constatant une répartition à peu près égale des pays audessus et en-dessous de la diagonale principale du graphique. De part et d'autre, les écarts à cette diagonale ne sont d'ailleurs pas considérables; ils n'avoisinent les dix points que pour une minorité de pays, et restent très en-deçà pour les autres.

En revanche les écarts entre pays sont, eux, très importants. Parmi les 45 ans et plus, on trouve par exemple $50 \%$ d'exposés à Chypre et $13 \%$ aux Pays-Bas. Ce qui nous intéresse ici est de voir comment se classent les pays, au regard de leurs "performances " sur l'emploi des seniors. On repère la présence du Royaume-Uni ou du Danemark (à fort taux d'emploi des seniors) parmi les pays où le niveau d'ensemble des exigences posturales semble modéré; et celle de la Hongrie (où ce taux est très faible) parmi les pays «à postures difficiles ». Mais la liaison n'est pas systématique. Globalement, le coefficient $\rho$ de corrélation des rangs entre les contraintes posturales des jeunes et l'emploi des seniors est de $-0,25$, donc dans le sens attendu quoique peu significatif - seuil de $21 \%$ selon la table des valeurs critiques du $\mathrm{r}$ de Bravais-Pearson, à $23(=25-2)$ degrés de liberté ; ce seuil signifie qu'un tel coefficient de corrélation aurait $21 \%$ de chances d'être atteint « par hasard ». La liaison avec les contraintes posturales des âgés est plus nette: $\rho=-0,32$ (seuil de $11 \%$ ). 
Figure 2. Salariés exposés à des postures douloureuses ou fatigantes (chez les moins de 45 ans et les 45 ans et + ), selon les pays en Europe (salariés de l'UE des 25,2005 )

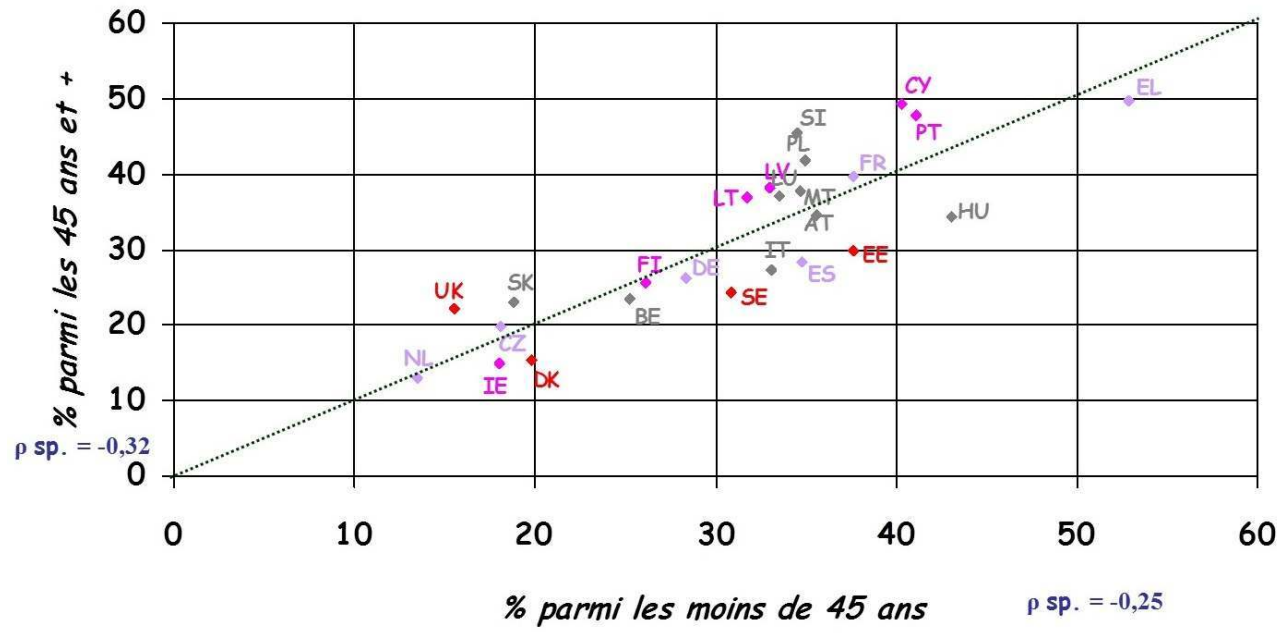

Code couleurs : taux d'emploi des 55-64 ans bas, assez bas, assez élevé, élevé

L'analyse statistique ne permet pas d'aller plus loin dans l'interprétation de ce premier ensemble de résultats, mais on peut s'appuyer sur d'autres sources de connaissances pour formuler les présomptions suivantes :

- Comme l'avaient montré des analyses longitudinales (Volkoff et coll., 1992 ; Molinié, 1999), l'exposition aux postures pénibles est une contrainte «durable» dans les parcours professionnels des salariés : «durable » d'une part en ce sens qu'elle ne recule pas d'une génération à une autre, mais aussi « durable » pour chaque personne ; un salarié peut ne pas être exposé, mais s'il l'est, il aura peu de possibilités de s'en écarter au cours de sa vie active. Les aménagements locaux et réaffectations diverses, que l'on a évoqués plus haut, sont précieux, mais leur portée est numériquement limitée.

- Dans les dernières années, en revanche, une forme de mise à l'abri consiste à quitter précocement la vie professionnelle, notamment pour les salariés souffrant de troubles articulaires (Derriennic et coll., 2003). On peut lire ainsi le recul des postures douloureuses après 55 ans. Dès lors, il n'est pas étonnant que les pays où le niveau global des contraintes posturales est relativement modéré soient aussi ceux où la vie professionnelle se prolonge davantage.

\section{L'abri (encore) possible vis-à-vis des horaires décalés}

De nombreuses études, sur le plan international, ont montré que la proportion de salariés en horaires décalés diminue après le milieu de carrière (Molinié, 2003). Dans la typologie des contraintes de travail déjà évoquée, les horaires décalés relevaient de la catégorie des « stationnaires-sélectives ». À long terme leur ampleur ne recule pas, mais une partie (de l'ordre de la moitié) des salariés concernés quittent ce type d'horaire au bout de dix à vingt ans d'exposition, et de jeunes salariés prennent la relève. C'était vrai en tout cas jusqu'à ces dernières années. On ignore si le contexte démographique et « l'érosion » des 
horaires réguliers diurnes (Bouffartigue et Bouteiller, 2002) vont permettre la poursuite de ces réaffectations.

Ici aussi l'état de santé joue un rôle. Les "postés » présentent moins de maladies que les salariés de jour aux mêmes âges, mais cela relève d'un mécanisme de sélection: pour tenir dans ces horaires, il faut disposer d'une santé robuste. Les troubles les plus graves sont perceptibles chez les anciens postés revenus en horaires de jour (Brugère et coll., 1997). Cependant de nombreux « petits » troubles, comme la dégradation du sommeil, la lassitude, ou la prise de poids, sont visibles chez les postés d'âge moyen, et se développent sous l'effet conjoint de l'avance en âge et des horaires décalés (ibid.). Ces constats chiffrés s'articulent avec les conclusions des physiologistes et des psychologues (Quéinnec, Gadbois, Prêteur, 1995). En particulier, la qualité du sommeil évolue avec l'âge. Le sommeil est moins bien structuré, moins ajustable aux changements temporels - et donc, moins réparateur dans les situations d'horaires atypiques.

Avancer en âge, c'est aussi connaître des conditions de vie familiale et sociale différentes, en fonction desquelles des horaires décalés n'ont pas les mêmes effets. L'âge des enfants, par exemple, influence l'horaire de présence à domicile, donc l'heure à laquelle la famille peut se retrouver. Le maintien durable de l'un ou l'autre conjoint - ou des deux - en horaires atypiques peut aussi faire apparaître des difficultés, dans la vie quotidienne ou les relations affectives, qui s'amplifient au fil des années, et finissent par rendre cette situation intolérable.

Dans l'enquête européenne, la sélection sur l'âge en ce domaine est manifeste. On se centrera sur le travail de nuit, que les études citées décrivent comme très pénalisant pour une partie des âgés. La question retenue est: "Habituellement, combien de fois par mois travaillez-vous durant la nuit, pour au moins une durée de deux heures entre 10 heures du soir et 5 heures du matin? » Pour des raisons de puissance statistique (les travailleurs de nuit étant très minoritaires), on regroupe toutes les personnes ayant indiqué un nombre non nul. Les variations selon l'âge en Europe sont représentées ci-dessous :

Figure 3. Au moins une nuit travaillée par mois, selon l'âge (salariés de l'UE des 25,2005 )
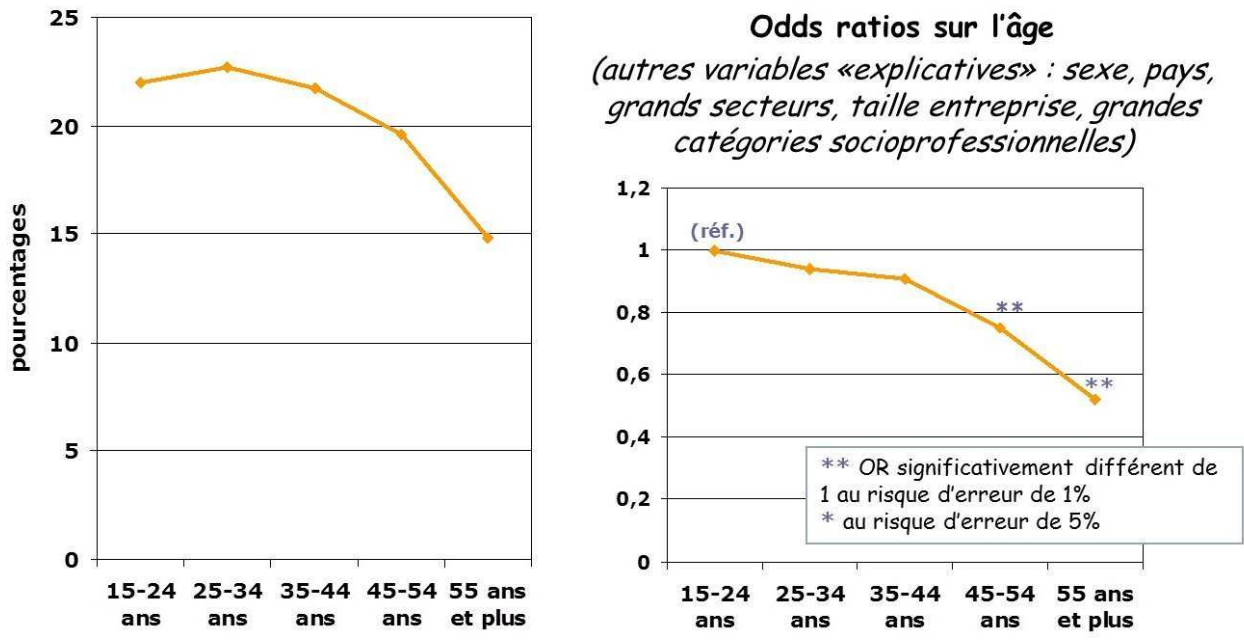

La tendance en 2005 est nette, tant en données brutes qu'après régression multivariée : la baisse du travail de nuit avec l'âge, surtout après 45 ans, est confirmée. Et ce constat vaut 
pour la plupart des pays, comme le montre la figure suivante. La quasi-totalité des pays sont représentés par des points se situant sous la diagonale, certains (dont la France) se plaçant très en-dessous; dans tous ces pays le travail de nuit est nettement plus répandu chez les jeunes que chez les âgés.

Le lien avec le taux d'emploi des seniors est alors logique: on ne constate pas de corrélation avec l'exposition des jeunes $(\rho=-0,04)$, mais un lien avec celle des âgés, lien d'intensité moyenne et dans le sens attendu ( $\rho=-0,29$, seuil de signification $16 \%)$. On voit, par exemple, que dans plusieurs pays d'Europe centrale (République tchèque, Pologne, Slovaquie) à faible taux d'emploi des seniors, le travail de nuit chez les 45 ans et plus est relativement répandu. À l'inverse, ce taux d'emploi est élevé dans des pays où le recours au travail de nuit est globalement modéré comme au Danemark, ou dans d'autres qui disposent d'une capacité suffisante de réaffecter les âgés en horaires diurnes comme en Estonie ou en Suède (nous interprétons ainsi la proportion nettement moindre des salariés en horaires nocturnes après 45 ans dans ces deux pays : par exemple, en Suède, $23 \%$ d'horaires nocturnes chez les jeunes, $13 \%$ chez les âgés; cependant, seule une analyse longitudinale assurerait qu'il s'agit bien de réaffectations).

Figure 4. Salariés exposés au travail nocturne (chez les moins de 45 ans et les 45 ans et +) selon les pays en Europe (salariés de l'UE des 25, 2005)

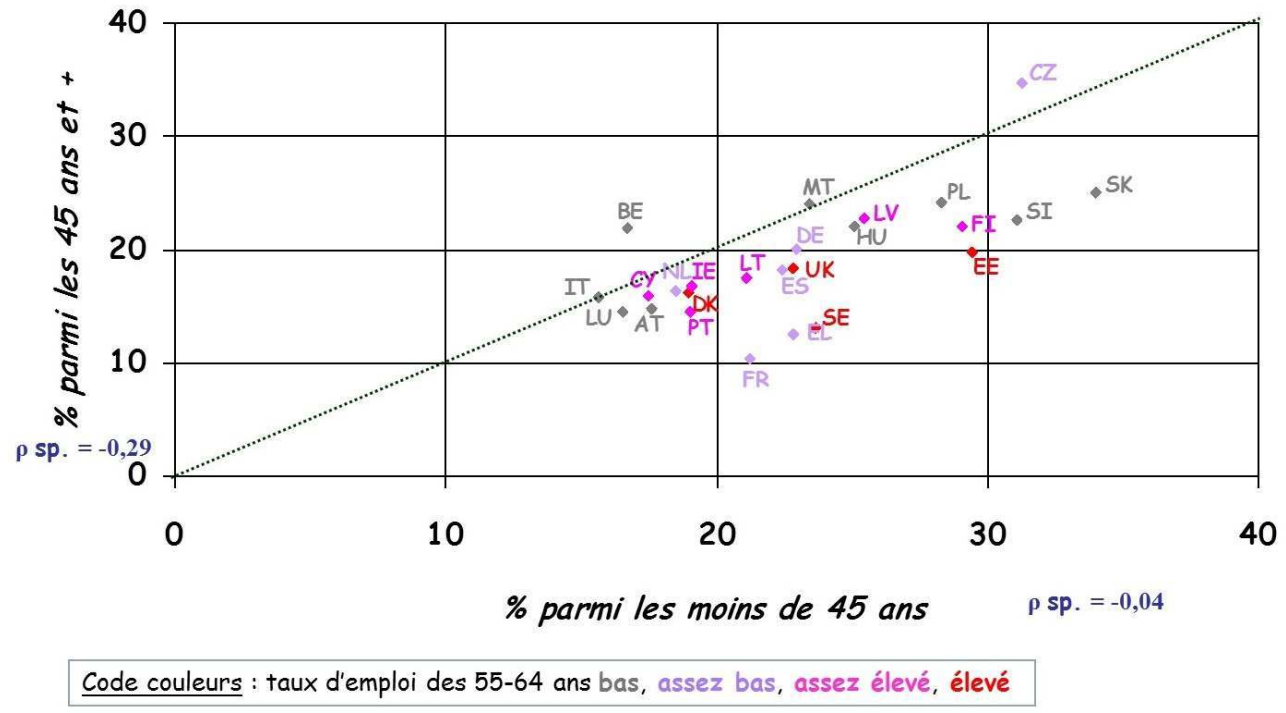

\section{Le rôle complexe de la pression temporelle dans le travail}

Dans le contexte général d'une intensification du travail dans les pays industrialisés, de nombreuses études (Askenazy et coll., 2006) signalent et analysent une accentuation du sentiment de hâte dans l'activité de travail. Au regard du vieillissement de la population en emploi, cette évolution pose problème.

Des études démographiques (Molinié, 1999, 2001b) ont en effet montré que les activités exercées sous contraintes de temps multiples, spécialement si elles sont répétitives, sont prioritairement assignées aux jeunes. De leur côté, les physiologistes et psychologues du travail indiquent une tendance au ralentissement avec l'âge dans l'exécution de gestes 
délicats, ou dans des prises de décision compliquées et instantanées, en raison surtout des attitudes de prudence et de vérification adoptées par les plus âgés, même dans des épreuves brèves. Sur cette question, les ergonomes aboutissent à des jugements nuancés, en examinant l'effet combiné des modifications de comportement liées à l'âge, et de la construction de l'expérience: des formes originales et efficientes de gestion des contraintes de temps apparaissent plus développées chez les âgés/expérimentés (Cloutier et coll., 1999; Volkoff, Pueyo, 2005b). Cependant ces stratégies sont plus ou moins réalisables selon les contextes. Selon une analyse issue de l'enquête française SVP50 (enquête interprofessionnelle sur la Santé et la Vie Professionnelle après 50 ans, réalisée par des médecins du travail en 2003 auprès de 11000 salariés),

« une pression temporelle forte pose de sérieux problèmes à la plupart des salariés quinquagénaires qui s'y trouvent exposés. Quatre-vingt pour cent d'entre eux jugent cette contrainte difficile. Ceux-ci présentent systématiquement des troubles plus fréquents, dans la plupart des domaines de la santé physique ou psychique » (Mardon, Volkoff, 2008).

La question la plus proche de cette thématique, dans l'enquête européenne, est la suivante : "Pouvez-vous me dire si votre travail nécessite de travailler selon des délais très stricts et très courts? » Comme ci-dessus pour les postures, les réponses possibles sont graduées par fréquence croissante, et l'on regroupe ici celles qui rendent compte d'une présence de cette contrainte « la moitié du temps » ou davantage.

Si l'on excepte la tranche d'âge des moins de 25 ans, numériquement peu nombreuse, la tendance à une baisse avec l'âge est confirmée, même si les écarts ne sont pas considérables (figure ci-dessous).

Figure 5. Travail en " délais très courts ", selon l'âge (salariés de l'UE des 25, 2005)

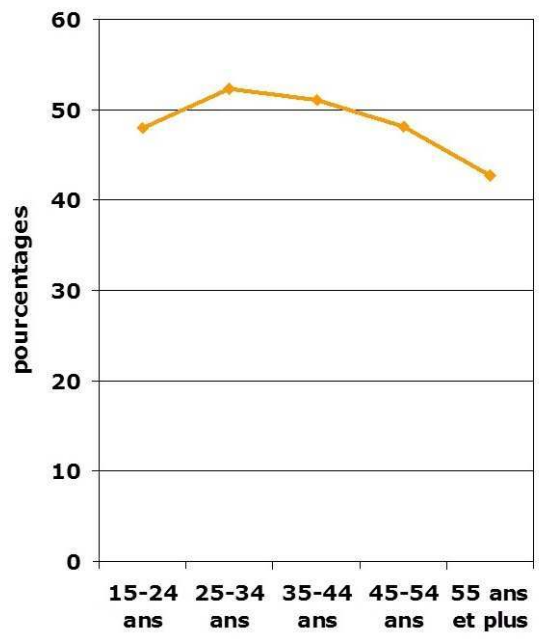

Odds ratios sur l'âge (autres variables «explicatives»: sexe, pays, grands secteurs, taille entreprise, grandes catégories socioprofessionnelles)

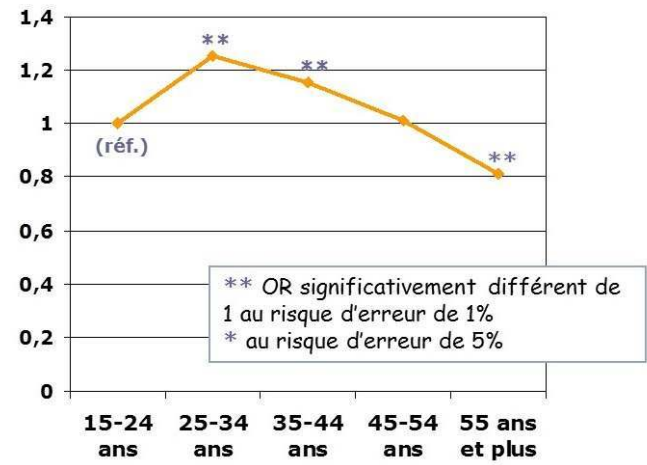

Dans la plupart des pays, le même mécanisme est à l'œuvre : la nécessité de travailler en délais courts est plus répandue avant 45 ans, ce qui place la quasi-totalité des points dans la moitié sud-est du graphique (figure suivante - pour clarifier le graphique, l'origine des axes est placée à $20 \%$ ). On voit notamment que dans certains pays baltes, ou méditerranéens, l'écart entre les deux groupes d'âge est supérieur à dix points.

L'examen des liens avec le taux d'emploi des seniors aboutit à un résultat moins attendu. La corrélation des rangs, entre emploi des seniors et «délais courts» dans le travail, 
ressort comme positive. Le lien est faible dans le cas des contraintes des 45 ans et plus ( $\rho=$ $+0,15)$, plus marqué s'agissant de l'exposition des jeunes $(\rho=+0,33$, significatif au seuil de $10 \%)$.

Figure 6. Salariés exposés au travail en " délais très courts " (dans l'ensemble et chez les 45 ans et + ), selon les pays en Europe (salariés de l'UE des 25, 2005)

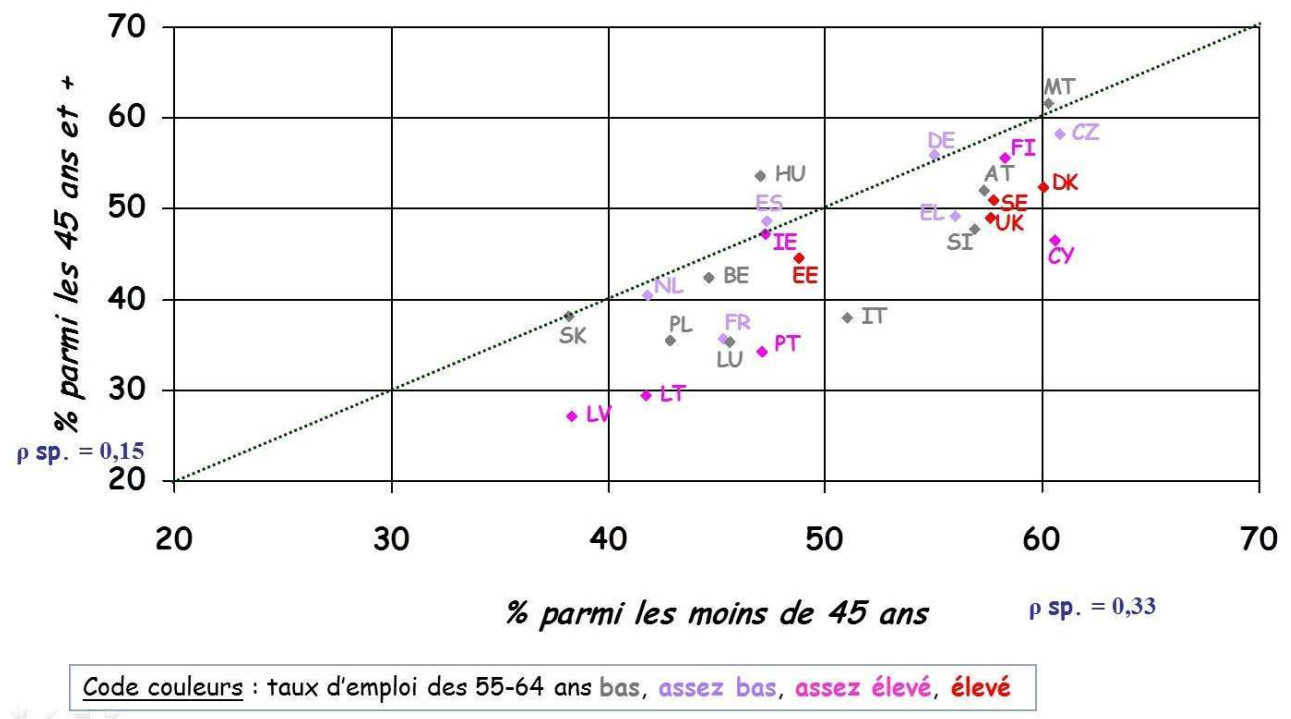

Autrement dit : les pays où l'emploi des seniors est le plus développé ne présentent pas spécialement des niveaux faibles en matière d'exposition moyenne à la pression temporelle (ce serait même plutôt l'inverse) ; mais dans le peloton de tête pour l'emploi des seniors on trouve souvent des pays où les jeunes salariés sont "surexposés » - ou, ce qui revient au même, des pays où est favorisé un retrait partiel des plus âgés, vers des situations de travail moins exposées à la pression. Au Danemark, en Suède, au RoyaumeUni, les « délais courts ", globalement répandus, le sont davantage chez les jeunes; au Portugal, et surtout en Lituanie ou Lettonie, le niveau global est inférieur mais là aussi la sélection sur l'âge est forte. Une surexposition des jeunes à la pression temporelle apparaitrait ainsi parmi les conditions - ou les conséquences - d'un taux élevé d'emploi des seniors. On peut synthétiser ce constat en classant les pays selon «le degré de sélection sur l'âge ", caractérisé par la différence : [ \% d'exposés chez les moins de 45 ans] - [ \% chez les 45 ans et +]. Le rapport de corrélation entre ce classement et celui des taux d'emploi de seniors est de 0,13 . Le lien n'est donc pas très net, mais les pays à fort taux d'emploi des seniors seraient plutôt ceux dans lesquels la sélection sur l'âge, au regard du « travail en délais courts », serait davantage marquée.

\section{Les ressources du « lifelong learning »}

Les phases de changement dans la vie professionnelle - qu'il s'agisse de modifier les techniques, les produits ou services, l'organisation, les objectifs de travail, ou qu'il s'agisse aussi de mobilité professionnelle ou polyvalence - impliquent d'acquérir ou restructurer des connaissances et des savoir-faire. Ces phases peuvent induire ou révéler des fragilités chez les anciens (Czaja et coll., 1989 ; Marquié et coll., 1994). Les difficultés qu'ils sont censés rencontrer en situation d'apprentissage ou de changement dans le 
travail constituent d'ailleurs une caractéristique négative aux yeux de beaucoup d'employeurs, ce qui contribue aux difficultés d'embauche des quinquagénaires (RichetMastain et Brunet, 2002) et à une relative mise à l'écart des âgés dans ces situations en entreprises, le changement impliquant davantage les jeunes (Molinié, 2001b; Behaghel, 2006). On perçoit aussi des réticences chez une partie des salariés âgés eux-mêmes. Dans les phases de "modernisation", certains ont des craintes pour leur emploi, d'autres éprouvent de l'inquiétude à l'idée d'endommager de nouveaux matériels, ou de ne pas maitriser un processus. D'autres encore ont une appréhension face aux situations d'apprentissage et à la mise en concurrence avec les plus jeunes à cette occasion (Delgoulet et Marquié, 2002).

41 L'avancée en âge peut s'accompagner de moins bonnes performances pour des fonctions cognitives élémentaires, fortement sollicitées en période d'apprentissage, comme la mémoire immédiate. Cependant, à l'échelle de la vie active, ces différences entre âges sont modérées, variables selon les individus, et elles dépendent beaucoup de la formation initiale ou continue - en particulier, de la qualité et de la régularité des formations professionnelles en cours de carrière. En outre, des compétences se sont élaborées dans le travail même, et constituent un atout en situation de changement, si ce changement est conduit de façon que chacun puisse bien comprendre en quoi ses compétences antérieures seront mobilisables (Delgoulet, 2001).

L'obligation de se confronter plus ou moins souvent à des situations nouvelles ne fait pas partie des domaines explorés par l'enquête européenne. Nous avons opté pour la question suivante : "Votre travail implique-t-il ou non d'apprendre des choses nouvelles?", en retenant les réponses affirmatives. La formulation en termes d'apprentissage, d'acquisition, nous écarte certes d'une analyse en matière de changements contraints. Un premier constat, cependant, va dans le sens des résultats précédents (figure ci-dessous): les " apprentissages de choses nouvelles" se font moins fréquents quand l'âge avance. La variation est nettement plus forte quand on procède à une analyse multivariée plutôt qu'en données brutes, en raison notamment des effets de structure sociale : la proportion de cadres croît avec l'âge, et par ailleurs cette catégorie mentionne des niveaux élevés de réponses positives; cette particularité gomme en partie les écarts entre âges, toutes catégories confondues; à catégorie "égale par ailleurs ", les écarts sont donc plus nets que dans les comparaisons simples. 
Figure 7. Avoir un travail qui implique d'apprendre des choses nouvelles, selon l'âge (salariés de I'UE des 25, 2005)

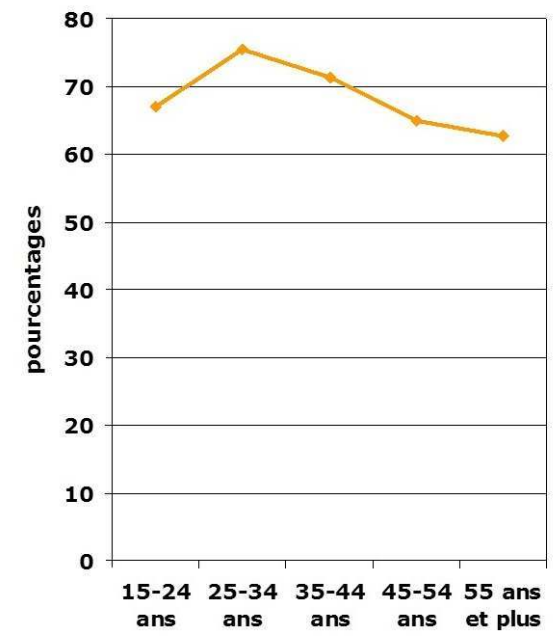

Odds ratios sur l'âge

(autres variables "explicatives»: sexe, pays, grands secteurs, taille entreprise, grandes catégories socioprofessionnelles)

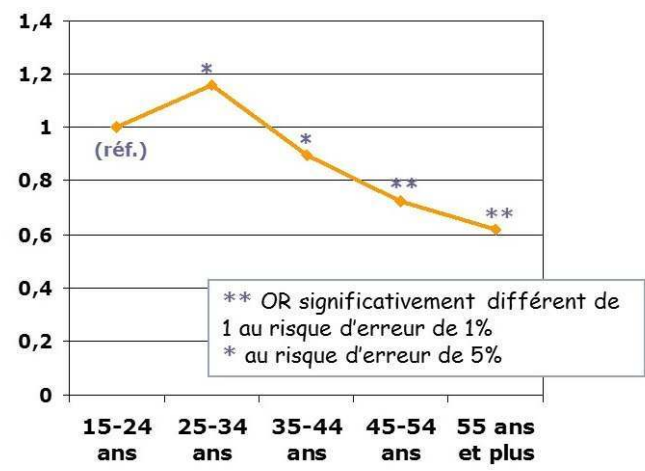

Pour clarifier le graphique, l'origine est placée à 40 \% sur chaque axe

Ce différentiel entre âges se retrouve dans la plupart des pays (figure suivante). Dans les autres, les pourcentages de réponses positives des jeunes et des âgés sont voisins. Quant aux différences entre pays, elles n'indiquent pas que les pays à fort emploi des seniors limiteraient les situations d'apprentissage. Au contraire, l'ampleur du «travail qui implique d'apprendre des choses nouvelles » est corrélée positivement avec le taux d'emploi des seniors : $\rho=+0,18$ pour le lien avec le classement sur les réponses des 45 ans et plus, et $\rho=+0,30$ (seuil de l'ordre de $15 \%$ ) pour celui des plus jeunes.

Figure 8 Salariés exposés au travail en " délais très courts » (dans l'ensemble, et chez 45 ans et +), selon les pays en Europe (salariés de l'UE des 25, 2005)

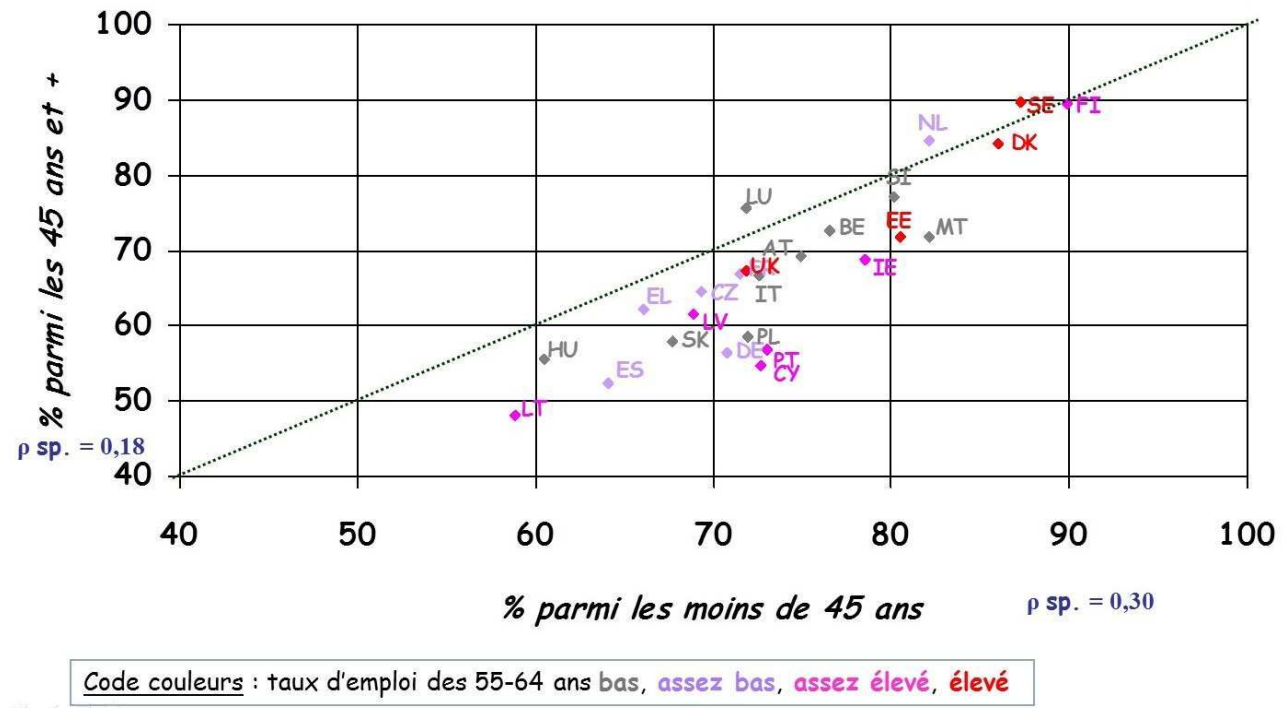

Avec les données dont nous disposons, il n'est pas possible de savoir si une question formulée dans des termes qui caractériseraient davantage une contrainte, aboutirait aux mêmes résultats - au vu de la littérature précédemment citée on peut penser que non. À 
titre indicatif, en effectuant les calculs sur la question concernant la rotation des tâches, on aboutit à une corrélation positive mais moins forte $(0.26)$ avec les réponses des jeunes, et à peu près nulle $(0,01)$ avec celle des âgés. Notons ici aussi que la question ne suppose pas que la rotation soit obligatoire.

Quoi qu'il en soit, le résultat présenté ici va dans le sens des modèles ergonomiques fondés sur une approche développementale de la vie professionnelle (Gaudart et WeillFassina, 1999), et des modèles sociologiques qui rapprochent les enjeux de durée de vie professionnelle et ceux liés à la formation tout au long de la vie (Guillemard, 2003).

On peut renforcer cette analyse en examinant une autre question, plus éloignée encore de l'idée de contrainte : «À quel point êtes-vous d'accord ou pas avec l'affirmation: au travail, j'ai des opportunités d'apprendre et d'évoluer? ", en retenant les réponses « d'accord » ou « tout à fait d'accord ", regroupées. Nous ne présenterons pas ces résultats en détail, mais retenons que les pays qui ont les taux d'emploi des seniors les plus élevés (pays baltes exceptés, donc essentiellement les pays nordiques ou britanniques) présentent des proportions de réponses positives supérieures à $66 \%$ chez les jeunes, à $56 \%$ chez les âgés. À l'opposé, les pays d'Europe centrale, mal classés pour l'emploi des seniors (Pologne, Hongrie, Slovénie, Slovaquie, République tchèque), présentent moins de $45 \%$ de réponses positives dans l'une et l'autre catégorie d'âge. En d'autres termes, dans chaque pays les "opportunités d'apprendre et d'évoluer» sont plus importantes en début de vie professionnelle - ce qui n'est pas surprenant - mais les pays dans lesquels ces " opportunités » originelles sont fortes (même si elles baissent ensuite) sont aussi ceux dans lesquels les seniors au travail sont nombreux.

\section{Maîtriser ou aménager les évolutions du travail}

Les relations que l'on a présentées, spécialement celles qui unissent le taux d'emploi des seniors dans un pays et certaines caractéristiques des conditions de travail ou des parcours professionnels, ne sont pas toutes très marquées. On retient tout de même que globalement les mécanismes sélectifs sur l'âge, en liaison avec les quatre caractéristiques étudiées, se maintiennent, même s'ils n'ont pas toujours l'ampleur constatée dans des enquêtes précédentes. On remarque surtout qu'un taux d'emploi élevé des seniors dans un pays va de pair avec: un niveau modéré de contraintes posturales à tout âge, une exposition différenciée des jeunes et des âgés (au bénéfice de ces derniers) au travail de nuit et à la pression temporelle et d'importantes possibilités d'apprentissage tout au long de la vie professionnelle.

Dans leur ensemble, ces résultats soulèvent à notre avis trois grandes interrogations, auxquelles ces données par elles-mêmes ne peuvent répondre :

1. Dans quelle mesure les quatre «exigences du travail » étudiées ici sont-elles globalement préjudiciables aux salariés concernés, et à ce titre devraient faire l'objet de politiques visant à les limiter, indépendamment de l'âge ? La réponse laisse peu de doutes en ce qui concerne les postures pénibles qui constituent une nuisance par elles-mêmes. C'est vrai en partie aussi pour le travail de nuit, même si les compensations dont il peut s'assortir (rémunération, temps de repos, type de responsabilité, ambiance de travail) sont parfois appréciées par les salariés concernés (Gadbois, 2004). La pression temporelle au travail, elle, a des effets contrastés - ce que d'ailleurs nos résultats confirment ; cela dépend des moyens de travail (informations, équipements, coopérations, objectifs de travail...) dont elle s'accompagne, et des résultats de cet ensemble en ce qui a trait à la qualité du travail réalisé (Volkoff, 2008). 
Quant à l'impératif d'apprendre des choses nouvelles, on ne peut pas le cataloguer du côté des nuisances, sauf si ses modalités de mise en œuvre sont mal conçues.

2. Les relations entre conditions de travail et emploi des seniors sont-elles directes? On pourrait en effet penser qu'elles reflètent pour l'essentiel les différences internationales en matière de structure sociale. En gros, les pays où la proportion de cadres est élevée auraient à la fois : de «meilleures" conditions de travail - moins de postures difficiles, d'horaires nocturnes; des dynamiques professionnelles plus vivaces, car les carrières des cadres sont moins figées; et un fort taux d'emploi des seniors parce que les cadres connaissent relativement peu de départs précoces. Cette interprétation est plausible. Elle est délicate à vérifier avec l'enquête utilisée ici: sur la base d'un millier d'individus par pays, les croisements âge x CS x caractéristiques du travail seraient très imprécis. En outre, on se trouverait face au problème bien connu de la comparabilité des nomenclatures sociales entre pays. Notre parti pris est de considérer qu'un éventuel (et probable) effet de structure sociale ne réfute pas nos principales conclusions : il y a bien un lien entre emploi des seniors et conditions de travail de la main-d'œuvre salariée, que ce lien passe ou non par la distribution des qualifications et les possibilités de promotion professionnelle.

3. Dans quel sens ces relations opèrent-elles? La culture ergonomique qui marque notre recherche nous a menés à affirmer, plus ou moins explicitement, que l'âge et les modalités de cessation d'activité « résultent » en partie des politiques du travail et de la formation. On peut défendre la thèse inverse, et certains s'y emploient (D'Autume et coll., 2006) : les dispositifs de retraite détermineraient un « horizon de carrière », qui lui-même délimiterait le cadre des aménagements des conditions de travail et des apprentissages. Plus cet «horizon » serait rapproché (en France, par exemple), moins les politiques du travail seraient actives. Cette thèse fait l'objet d'un débat entre économistes (voir par exemple Blanchet, 2006 ; Benallah et coll., 2008), dans lequel nous n'avons pas vocation à intervenir. Disons seulement que, d'après notre connaissance concrète des pratiques d'entreprises dans le domaine du travail, celles-ci relèvent peu des politiques publiques dans le domaine de la retraite, même si l'inflexion de ces dernières devrait mettre à l'épreuve les "impératifs " d'organisation des entreprises, dont dépendent les caractéristiques du travail (comme les contraintes posturales, le travail nocturne ou la pression temporelle) et les modes d'apprentissage. En France, par exemple, il y a depuis le $1^{\text {er }}$ janvier 2010 obligation de négocier un accord d'entreprise notamment sur l'emploi des seniors; les pratiques qui en découleront mériteront d'être examinées avec attention. Plus généralement, la question des liens entre pratiques d'entreprise et politiques publiques dans ce domaine mériterait d'être directement étudiée en sciences de la gestion. Comme nous l'indiquions au début de cet article, cela ne revient pas à nier les effets des politiques de retraites - ni d'ailleurs, ceux des autres aspects du modèle social d'un pays, comme le taux d'emploi global ou les profils de carrière salariale - sur les comportements de départ ou de maintien dans l'emploi.

49 Si donc on admet que les grandes caractéristiques du travail étudiées dans cet article (postures corporelles, horaires décalés, pression du temps, changements importants dans le travail) constituent bien des enjeux majeurs au regard de la durée de vie professionnelle, une remarque s'impose : ce sont des caractéristiques en expansion. En France, par exemple, le pourcentage de salariés déclarant devoir « rester longtemps dans une posture pénible », est en progrès à long terme (Bué et coll., 2007) ; ni la mécanisation, ni l'expansion des emplois tertiaires n'ont résorbé cette difficulté. Le travail de nuit augmente aussi, progressivement (ibid.) et, toujours à long terme, on constate une expansion de toutes formes de contraintes temporelles (ibid.). Quant aux changements de techniques et d'organisation, on sait qu'ils ont connu une nette accélération à partir du milieu des années 90 (Greenan et Mairesse, 2006). 

comparées - que les pays " performants » en matière d'emploi des seniors ont su, mieux que les autres, maîtriser ou aménager ces évolutions: en évitant l'expansion des sollicitations posturales; en offrant aux salariés travaillant de nuit ou sous fortes contraintes de temps des possibilités de s'écarter de ces contraintes en cours de vie active; et en créant les conditions d'une approche "vertueuse " des changements, porteuse d'opportunités d'apprendre et non de remise en cause brutale des acquis de l'expérience. Bien entendu, ces spécificités nationales entretiennent des liens avec les structures productives et sociales des différents pays, et ces liens eux-mêmes mériteraient d'être étudiés. fréquentes des politiques d'emploi, de travail et de formation entre pays offrent une nouvelle occasion de s'attaquer à ces enjeux. Certes, la plupart des recommandations que l'on peut formuler ne sont pas "spécifiques aux seniors ». L'amélioration d'ensemble des conditions de travail reste une voie sûre pour éviter de mettre les âgés en difficulté, et aussi pour éviter l'usure précoce des jeunes. Cependant, des mesures dédiées à la population des "seniors", en particulier ceux dont la santé est déficiente, ont leur légitimité, et plusieurs pays européens y font largement appel. Cornilleau et coll. (2007) rappellent ainsi que «en Finlande, les dépenses d'invalidité représentent 3,5\% du PIB contre 1,5\% en France et 2,5\% dans l'UE15»; ces dépenses ne sont pas toutes consacrées aux seniors, mais elles jouent un rôle important dans les modalités de fin de vie active.

outre, la prise en compte de l'âge est importante pour une autre raison, qui nous intéresse davantage ici : elle rappelle que les salariés sont, simplement, différents les uns des autres. Ces différences s'accentuent quand le parcours de vie, et avec lui le parcours professionnel, s'allongent. La question est alors de concevoir des situations de travail et d'apprentissage où ces personnes différentes peuvent trouver leur place, et tirer profit de leurs expériences, elles-mêmes diverses. Cela suppose de ne pas imposer aux hommes et aux femmes au travail des contraintes extrêmes (efforts trop violents, horaires trop fatigants, urgences trop fréquentes...), d'aménager pour certains des modes de sortie adaptés à leur santé et à leur itinéraire professionnel, mais aussi de donner aux salariés expérimentés les moyens d'élaborer et d'adopter des façons de travailler qui leur conviennent.

\section{Encadré 1. L'enquête européenne sur les conditions de travail (EWCS)}

Cette enquête est réalisée à l'initiative de la Fondation européenne pour l'amélioration des conditions de vie et de travail, sise à Dublin. Sa périodicité est quinquennale. L'édition 2005, à laquelle cet article fait appel, est la quatrième. Trente et un pays étaient concernés, mais seuls 25 sont retenus ici, en écartant des pays où l'enquête était réalisée pour la première fois, avec une méthodologie sans doute moins bien stabilisée. Dans la plupart des pays l'échantillon est de l'ordre de 1000 actifs ; il est un peu moindre dans quelques pays de plus petite taille.

L'ensemble de l'échantillon compte $82 \%$ de salariés, sur lesquels porte la présente étude. Les unités statistiques ont été pondérées à trois niveaux (taille du ménage, non-réponses, pays) selon les coefficients élaborés par les responsables de l'enquête.

Voir le site de l'enquête :

www.eurofound.europa.eu/ewco/surveys/index.htm 
Encadré 2. Les taux d'emploi des seniors en Europe (2005)

Les taux d'emploi des seniors (55-64 ans) dans les pays d'Europe font l'objet d'un suivi régulier, leurs niveaux sont bien connus et diffusés. Pour le présent article nous reprenons les niveaux mesurés par l'enquête européenne sur les forces de travail, d'Eurostat. Pour des analyses plus générales sur ces données, on peut par exemple consulter les documents présentés au Colloque du Conseil français d'orientation des retraites le 29 novembre 2007.

(http://www.strategie.gouv.fr/IMG/pdf/Dossierdu_participant29_nov_2007_-2.pdf) ; voir aussi Aliaga et Romans (2006). Le graphique ci-dessous présente le taux d'emploi des seniors en 2005 dans les 25 pays étudiés ici, classés par taux croissant. Les teintes utilisées, que l'on retrouvera dans des graphiques de l'article, permettent de visualiser quelques catégories de pays ; ce découpage tient compte des proximités et disparités constatées, mais les limites entre catégories n'ont pas de signification précise.

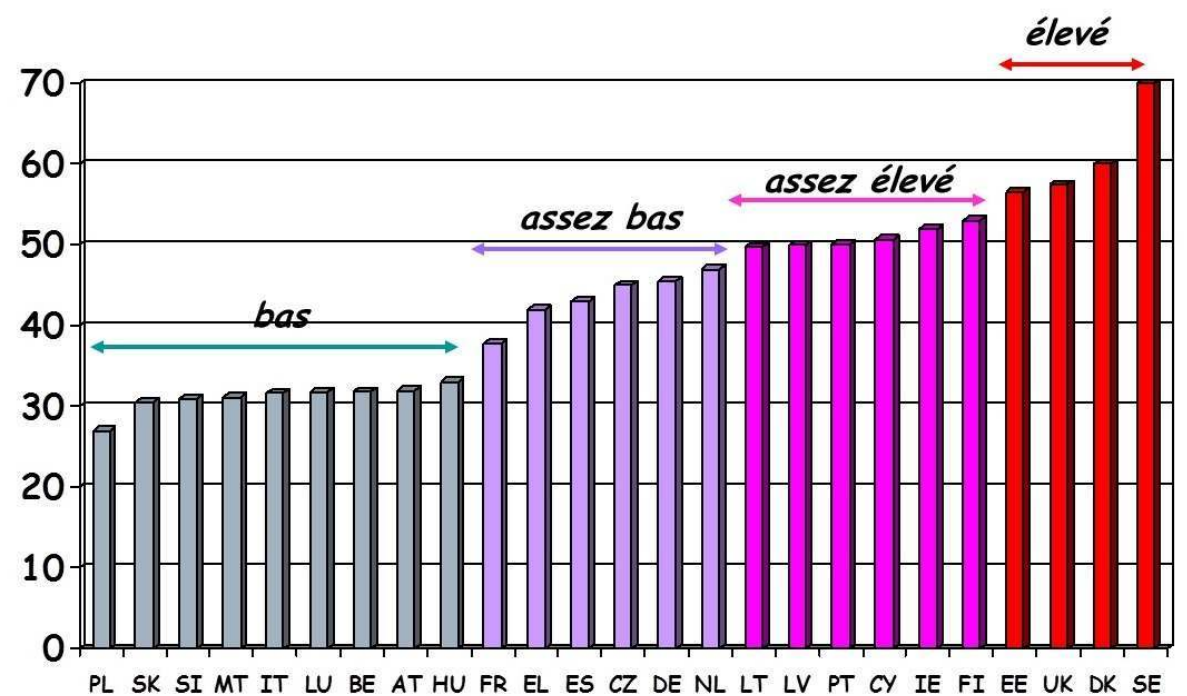

Signification des sigles

PL : Pologne

SK : Slovaquie

SI : Slovénie

MT : Malte

IT : Italie

LU : Luxembourg

$\mathrm{BE}$ : Belgique

AT : Autriche

$\mathrm{HU}$ : Hongrie

FR : France

EL : Grèce

ES : Espagne

$\mathrm{CZ}$ : République tchèque 

DE : Allemagne
NL : Pays-Bas
LT : Lituanie
LV : Lettonie
PT : Portugal
CY : Chypre
IE : Irlande
$\mathrm{FI}$ : Finlande
$\mathrm{EE}$ : Estonie
UK : Royaume-Uni
DK : Danemark
$\mathrm{SE}$ : Suède

\section{BIBLIOGRAPHIE}

Aliaga, C., Romans, F. (2006). L'emploi des seniors dans l'Union européenne. Statistiques en bref, Eurostat, $\mathrm{n}^{\circ} 15$.

Askenazy, P., Cartron, D., De Coninck, F., Gollac, M. (dir.) (2006). Organisation et intensité du travail, Octarès, Toulouse.

Behaghe,l L., (2006). Changement technologique et formation tout au long de la vie. Revue économique, 57, 6, www.inra.fr

Benallah, S., Duc, C., Legendre, F. (2008). Peut-on expliquer le faible taux d'emploi des seniors en France? Revue de l'OFCE, 105.

Blanchet, D. (2006). Âge ou distance à la retraite : quel est le principal déterminant de l'emploi des seniors ? Économie et statistique, 397.

Bouffartigue, P., Bouteiller, J. (2002). L'érosion de la norme du temps de travail, Travail et Emploi, 92, 43-55.

Bourget-Devouassoux, J., Volkoff, S. (1991). Bilans de santé des carrières d'ouvriers. Économie et statistique, 242, 83-93.

Brugère, D., Barrit, J., Butat, C., Cosset, M., Volkoff, S. (1997). Shiftwork, Age and Health : an Epidemiological Investigation. International Journal of Occupational and Environmental Health, 3, 3, 15-23.

Bué, J., Coutrot, T., Hamon-Cholet, S., Vinck, L. (2007). Conditions de travail : une pause dans l'intensification du travail. Premières informations premières synthèses $n^{\circ} s 1-2$, Dares, ministère du Travail.

Chassaing, K. (2004). Vers une compréhension de la construction des gestuelles avec l'expérience : le cas des «tôliers » d'une entreprise automobile. PISTES, 6, 1. https:// pistes.revues.org/3280 
Chia, K.S. (1997). Significant-itis - an obsession with the p-value, Scandinavian Journal of Work and Environmental Health, 23, 152-154.

Cloutier, E., David, H., Prévost, J., Teiger, C. (1999). Les compétences des auxiliaires familiales et sociales expérimentées dans la gestion des contraintes et des risques dans l'activité de travail. Formation Emploi, 67, 63-75.

Cornilleau, G., Sterdyniak, H., Allègre, G., Creel, J., Guillou, S., Levasseur, S., Mathieu, C., Reynès, F., Stancanelli, E., Touzé, V. (2007). Étude comparative sur les pays européens ayant un taux d'emploi des seniors élevé. OFCE, Rapport au Conseil d'orientation des retraites, septembre 2007. www.corretraites.fr/IMG/pdf/doc-1203.pdf

Czaja, S.J., Hammond, K., Blascovitch, J.J., Swede, H. (1989). Age related differences in learning to use a text-editing system. Behaviour and Information Technology, 8, 4.

D’Autume, A., Betbèze, J.P., Hairault, J.O. (2006). Les seniors et l'emploi en France. Rapport du Conseil d'analyse économique. www.cae.gouv.fr/spip.php?article38

David, H., Volkoff, S., Cloutier, E., Derriennic, F. (2001). Vieillissement, organisation du travail et santé. PISTES, 3,1. https://pistes.revues.org/3745

Delgoulet, C. (2001). La construction des liens entre situations de travail et situations d'apprentissage dans la formation professionnelle. PISTES, 3, 2. https://pistes.revues.org/3718

Delgoulet, C., Marquié, J.C. (2002). Age differences in learning maintenance skills : a field study. Experimental Aging Research, 28.

Delgoulet, C., Millanvoye, M., Volkoff, S. (2005). Les capacités des travailleurs vieillissants : une approche conditionnelle. Futuribles, 314, 5-23.

Derriennic, F., Saurel-Cubizolles, M.J., Monfort, C. (2003). Santé, conditions de travail et cessation d'activité des salariés âgés. Travail et Emploi, 96, 37-53.

Desriaux, F., Derriennic, F., Cassou, B., Lecuyer, G. (1987). Âge et changement de tâches dans une cohorte de salariés français d'une usine de construction mécanique. Le travail humain, 50, 3 , 225-236.

Gadbois, C. (2004). Les discordances psychosociales des horaires atypiques : questions en suspens. Le travail humain, 67, 1, 63-85.

Gaillard, A. (1995), À la recherche des postes sélectifs : travail et vieillissement dans une petite usine de confection. Dans J.-C. Marquié, D. Paumès, S. Volkoff, Le travail au fil de l'âge. Octarès, Toulouse, p. 131-155.

Gaudart, C. (2000). Conditions for maintaining ageing operators at work - a case study conducted at an automobile manufacturing plant. Applied Ergonomics, 31, 5, 453-462.

Gaudart, C., Molinié, A.-F., Pueyo, V. (2006). Du vieillissement à la diversité des âges au travail Questions pour l'ergonomie. In Actes du XLI ${ }^{\mathrm{e}}$ congrès de la SELF, Ergonomie et santé au travail (p. 471-476). Toulouse : Octarès (Coll. Le travail en débats). www.ergonomie-self.org/ documents/41eme-Caen-2006/session4.pdf.

Gaudart, C., Weill-Fassina, A. (1999). L'évolution des compétences professionnelles au cours de la vie professionnelle : une approche ergonomique. Formation Emploi, 67.

Gonon, O. (2003). Des régulations en lien avec l'âge, la santé et les caractéristiques du travail : le cas des infirmières d'un centre hospitalier français. PISTES, 5, 1. https://pistes.revues.org/3336

Gollac, M. (1998). À marches forcées. Mémoire d'habilitation à diriger des recherches sous la direction de J.-C. Combessie, Université de Paris 8. 
Greenan, N., Mairesse, J. (2006). Les changements organisationnels, l'informatisation des entreprises et le travail des salariés. Un exercice de mesure à partir de données couplées entreprises/salariés. Dans N. Greenan, J. Mairesse (s/d.), « Réorganisations, changements du travail et renouvellement des compétences », Revue économique, 57, 6, 1137-1175.

Guillemard, A.M. (2003). L'âge de l'emploi. Les sociétés à l'épreuve du vieillissement. Armand Colin, Paris, $286 \mathrm{p}$.

Hairault, J-O., Langot, F., Sopraseuth, T. (2007). Les effets à rebours de l'âge de la retraite sur le taux d'emploi des seniors. Économie et statistique, 397, 51-68.

Jolivet, A. (2002). La politique européenne en faveur du vieillissement actif. Retraite et Société, 36, 140-157.

Mardon, C., Volkoff, S. (2008). Les salariés âgés face au travail « sous pression ». Connaissance de l'emploi, 52, Centre d'études de l'emploi.

Marquié, J.-C., Thon, B., Baracat, B. (1994). Age influence on attitudes of office workers faced with new computerized technologies. Applied Ergonomics, 25, 3.

Merllié, D., Paoli, P. (2001). Ten Years of Working Conditions in the European Union. Fondation de Dublin.

Millanvoye M., 1993. Une préoccupation ergonomique : anticiper sur le vieillissement des salariés. Travail et sécurité, 519.

Millanvoye, M. (1995). Le vieillissement de l'organisme avant 60 ans. Dans J.-C. Marquié, D. Paumès et $\mathrm{S}$. Volkoff (Eds.), Le travail au fil de l'âge. Octarès, Toulouse, 175-209.

Molinié, A.-F. (1999). Professional Careers, Work Constraints, and Age-Related Selection : A Study on 21,000 Wage-Earners of Four Age Cohorts in 1990 and 1995. Experimental Aging Research, 25, 399-404.

Molinié, A.-F. (2001a). Parcours de travail et fins de vie active dans différentes générations. Centre d'études de l'Emploi, Quatre Pages, 45.

Molinié, A.-F. (2001b). Quelles contraintes pour quels âges ? Annales des Mines, mai, 57-61.

Molinié, A.-F. (2003). Âge et conditions de travail dans l'Union européenne, Dublin, Fondation européenne pour l'amélioration des conditions de vie et de travail, Dublin, Office des publications officielles des communautés européennes, 178 p. www.eurofound.europa.eu/pubdocs/2002/106/ fr/1/ef02106fr.pdf

Pacaud, S. (1975). Le travailleur vieillissant - Quelques réflexions sur ses difficultés, mais aussi ses facilités d'adaptation. Dans A. Laville, C. Teiger, A. Wisner (éds). Âge et contraintes de travail. NEB éditions scientifiques, Jouy-en-Josas, 115-179.

Pailhé, A. (2005). Les conditions de travail : quelle protection pour les salariés en France? Population - F, 1-2, 91-126.

Piaget, J. (1987). Les conduites de l'adulte. Dans J. Piaget, P. Mounoud, J.P. Bronckart (éds.), Psychologie. Paris, Gallimard (La Pléïade), 847-852.

Quéinnec, Y., Gadbois, C., Prêteur, V. (1995). Souffrir de ses horaires de travail : poids de l'âge et histoire de vie. Dans J.-C. Marquié, D. Paumès etS. Volkoff (Eds.), Le travail au fil de l'âge. Octarès, Toulouse, 277-304.

Richet-Mastain, L., Brunet, F. (2002). L'âge des salariés joue surtout à l'embauche. Premières informations et premières synthèses, 15,3 . 
Touranchet, A., Derriennic, F., De Stampa, M. (2001). Rôle de l'âge et des conditions de travail sur l'apparition et la disparition des troubles de la mobilité physique entre 1990 et 1995 dans l'enquête Estev. Dans B. Cassou (coord.), Travail, santé, vieillissement. Relations et évolutions. Octarès, Toulouse, 125-136.

Volkoff, S. (2001). Lire l'âge pour interpréter le travail. Annales des Mines, mai, 51-57.

Volkoff, S., Laville, A., Maillard, M.C. (1992). Âge et travail : contraintes, sélection et difficultés chez les 40-50 ans. Travail et Emploi, 54, 20-33.

Volkoff, S. (coord.), avec M.-F. Cristofari, C. Gadbois, A. Laville, A.-F. Molinié, S. PrunierPoulmaire, S. Stock, N. Vézina (2005a). L'ergonomie et les chiffres de la santé au travail : ressources, tensions et pièges. Octarès, Toulouse.

Volkoff, S., Pueyo, V. (2005b). How do Elderly Workers Face Tight Time Constraints ? In Costa, G., Goedhard, W., Ilmarinen, J. (eds), Assessment and Promotion of Ability, Health and Well-being of Ageing Workers, Elsevier ICS, 1280, 17-22.

Volkoff, S. (2008). L'intensification du travail « disperse » les problèmes de santé. Dans G., de Terssac, C., Saint-Martin, C., Thébault, La précarité : une relation entre travail, organisation et santé. Octarès, Toulouse.

\section{RÉSUMÉS}

L'emploi des âgés constitue une préoccupation croissante, notamment pour les institutions internationales. Cet article analyse les liens entre exigences du travail et emploi des seniors, dans les pays d'Europe. La réflexion proposée fait appel à l'ergonomie et à la démographie. Des études locales et des approches statistiques attirent en effet l'attention sur des difficultés, particulièrement sensibles chez les âgés, liées à quatre catégories d'exigences : postures pénibles, horaires décalés, pression temporelle élevée, ou changements rapides de techniques ou d'organisation. Pour chacune de ces quatre caractéristiques, l'article explique en quoi elles sont problématiques pour les âgés, puis interroge leur lien avec l'emploi des seniors dans 25 pays, à l'aide des données de l'enquête européenne sur les conditions de travail. Nos résultats suggèrent que les pays "performants" en termes d'emploi des seniors ont su, mieux que les autres, maîtriser ou aménager ces caractéristiques du travail, en particulier en limitant les sollicitations physiques et en favorisant les apprentissages tout au long de la vie professionnelle.

Employment rates of ageing workforce have become a major concern, in particular for international organisms. In this article we analyze the links between work constraints and employment of elderly, by comparing various European countries. Ergonomic and demographic methodologies are being called on. Local studies and statistical approaches actually emphasize difficulties, especially perceived by elderly workers, in relation with four main types of constraints: painful positions, shift work, high time pressure, and fast paced changes in techniques or organisation. For each of these characteristics, we first recap why it is tricky for elderly, then we study its relations with seniors' employment rate in 25 countries, using the ESWC data (European Survey on Working Conditions). Our results suggest that «high performance " countries in terms of senior employment have, better than others, mastered or adapted these characteristics, in particular by lowering physical efforts, and by promoting lifelong learning.

El empleo de adultos mayores constituye una preocupación creciente, notablemente para los organismos internacionales. Este artículo analiza los lazos entre las exigencias del trabajo y el 
empleo de adultos mayores en países de Europa. La reflexión propuesta requiere metodologías provenientes de la ergonomía y la demografía. Los estudios locales y los abordajes estadísticos atraen la atención sobre las dificultades, particularmente percibidas por trabajadores de edad avanzada, ligadas a cuatro categorías de exigencias : posturas dolorosas, horarios de trabajo, alta presión temporal y cambios rápidos en las técnicas o en la organización. Para cada una de las cuatro características, el artículo explica como éstas son problemáticas para las personas de edad avanzada y analiza su relación con el empleo de personas mayores en 25 países, con la ayuda de datos de la Encuesta Europea sobre condiciones laborales. Nuestros resultados sugieren que los países que emplean con éxito a personas mayores han sabido controlar o cambiar, mejor que otros países, las características del trabajo mencionadas. Particularmente, se han limitado las solicitaciones físicas y se ha favorecido el aprendizaje a lo largo de toda la vida profesional.

INDEX

Palabras claves : edad, envejecimiento, horarios, posturas, dificultades de tiempo, aprendizajes, entrenamientos, comparaciones internacionales

Keywords : age, ageing, schedules, positions, time constraints, training, international comparisons

Mots-clés : âge, vieillissement, horaires, postures, contraintes de temps, apprentissage, comparaisons internationales

\section{AUTEURS}

\section{CÉLINE MARDON}

celine.mardon@cee-recherche.fr, CREAPT - CEE, « le Descartes I », 29, promenade Michel Simon, 93166 Noisy-le-Grand Cedex

\section{SERGE VOLKOFF}

serge.volkoff@cee-recherche.fr, CREAPT - CEE, « le Descartes I », 29, promenade Michel Simon, 93166 Noisy-le-Grand Cedex 